\title{
Signaling Pathways Regulating Thermogenesis
}

\author{
Chihiro Tabuchi and Hei Sook Sul* \\ Department of Nutritional Sciences and Toxicology, University of California, Berkeley, Berkeley, CA, United States
}

Obesity, an excess accumulation of white adipose tissue (WAT), has become a global epidemic and is associated with complex diseases, such as type 2 diabetes and cardiovascular diseases. Presently, there are no safe and effective therapeutic agents to treat obesity. In contrast to white adipocytes that store energy as triglycerides in unilocular lipid droplet, brown and brown-like or beige adipocytes utilize fatty acids (FAs) and glucose at a high rate mainly by uncoupling protein 1 (UCP1) action to uncouple mitochondrial proton gradient from ATP synthesis, dissipating energy as heat. Recent studies on the presence of brown or brown-like adipocytes in adult humans have revealed their potential as therapeutic targets in combating obesity. Classically, the main signaling pathway known to activate thermogenesis in adipocytes is $\beta_{3}$-adrenergic signaling, which is activated by norepinephrine in response to cold, leading to activation of the thermogenic

OPEN ACCESS

Edited by:

Jiqiu Wang,

Shanghai Jiao Tong University, China

Reviewed by:

Andreas Hoeflich,

Leibniz Institute for Farm Animal Biology (FBN), Germany Rosalba Senese,

University of Campania Luigi Vanvitelli,

${ }^{*}$ Correspondence: Hei Sook Sul hsul@berkeley.edu

Specialty section: This article was submitted to Cellular Endocrinology, a section of the journal

Frontiers in Endocrinology

Received: 14 August 2020 Accepted: 25 February 2021 Published: 26 March 2021

Citation:

Tabuchi C and Sul HS (2021) Signaling Pathways Regulating Thermogenesis.

Front. Endocrinol. 12:595020. doi: 10.3389/fendo.2021.595020 program and browning. In addition to the $\beta_{3}$-adrenergic signaling, numerous other hormones and secreted factors have been reported to affect thermogenesis. In this review, we discuss several major pathways, $\beta_{3}$-adrenergic, insulin/IGF1, thyroid hormone and TGF $\beta$ family, which regulate thermogenesis and browning of WAT.

Keywords: thermogenesis, brown adipose tissue, browning/beiging, b3-adrenergic signaling, UCP1, insulin/IGF1 signaling, thyroid hormone, TGF $\beta$ superfamily

\section{INTRODUCTION}

Brown adipose tissue (BAT) is specialized in heat production through non-shivering thermogenesis by burning fuels such as fatty acids (FA) and glucose (1). BAT is crucial for maintaining body temperature especially for infants who have limited ability to perform shivering thermogenesis, due to underdeveloped skeletal muscle (2). In adults, BAT contributes to energy expenditure as indicated by an inverse correlation between the presence of active BAT and central obesity $(3,4)$. In addition to numerous mitochondria, BAT possesses multilocular lipid droplets that provide FAs for $\beta$-oxidation, which drives tricarboxylic acid (TCA) cycle and electron transport chain (ETC) to create proton gradient (5). In contrast, white adipose tissue (WAT) serves as an energy storage and has a unilocular lipid droplet and fewer mitochondria.

Of all tissues, BAT expresses the highest levels of uncoupling protein 1 (UCP1) and UCP1 plays a central role in non-shivering thermogenesis (6). UCP1 acts as a proton transporter in the inner membrane of mitochondria, which allows protons to leak from intermembrane space into the mitochondrial matrix (7). This uncouples the proton gradient from ATP synthesis, dissipating the proton gradient in the form of heat. Therefore, having a large number of mitochondria and multilocular lipid droplets enables BAT to maximize its thermogenic capacity. Although WAT normally has little UCP1 expression, some cells in subcutaneous WAT depots display thermogenic 
capacity with elevated UCP1 expression upon certain stimulations, the process so-called 'browning/beiging' (8). These thermogenic adipocytes are termed 'beige/brite' adipocytes, which seem to originate from different precursor cells than brown adipocytes, based on distinct gene signatures (9). However, transdifferentiation of mature white adipocytes to thermogenic beige adipocytes have also been reported (10). It is plausible that these thermogenic adipocytes can be generated by distinct mechanisms.

Classical brown adipocytes originate from a subset of multipotent stem cells in the dermomyotome that express engrailed 1 (EN1), paired box 7 (PAX7) and myogenic factor 5 (MYF5) (11). While the MYF5 ${ }^{+} / \mathrm{PAX}^{+} / \mathrm{EN1}^{+}$progenitor cells can give rise to other cell types, such as skeletal myocytes, they commit to brown adipocyte lineage upon early B cell factor 2 (EBF2) expression (12). Additionally, PR domain containing 16 (PRDM16) stimulates brown adipocyte differentiation over myoblast differentiation through the interaction with peroxisome proliferator activated receptor $\gamma$ (PPAR $\gamma$ ) (13). Ewing Sarcoma (EWS)/Y-box binding protein 1 (YBX1) complex promotes bone morphogenetic protein 7 (BMP7) transcription, which also determines brown adipocyte lineage during development (14). Committed brown precursor cells then differentiate into mature brown adipocytes through a series of transcriptional events $(11,15)$. ZFP516 stimulates thermogenic gene expression such as UCP1, DIO2 and CIDEA to promote brown adipocyte differentiation. ZFP516 interacts with not only PRDM16, but also with LSD1 in activating thermogenic gene program. ZC3H10 upregulates transcription of genes for mitochondrial biogenesis, such as NRF1 and TFAM $(16,17)$. EBF2 activates transcription of $\operatorname{PPAR} \gamma$, which promotes expression of numerous lipid metabolic genes, such as fatty acid binding protein (FABP4/aP2), cluster of differentiation 36 (CD36) and lipoprotein lipase (LPL) (18). In addition, EBF2 and PPAR $\gamma$ cooperatively activate transcription of PRDM16. CEBP $\beta$ also activates PPAR $\gamma$ transcription to promote adipogenic and thermogenic gene expression (19). PPAR $\gamma$ coactivator $\alpha$ $(\mathrm{PGC} 1 \alpha)$ is another transcriptional co-regulator that interacts with many proteins including PPAR $\gamma$, PRDM16 and IRF4 to promote BAT development, especially mitochondrial biogenesis through NRF1 and TFAM $(15,20)$. Some of the aforementioned transcription factors and co-activators such as ZFP516, ZC3H10 and PGC1 $\alpha$ also induce thermogenic gene expression upon $\beta_{3^{-}}$ adrenergic stimuli in mature brown and white adipocytes, the latter of which leads to browning.

Environmental cues such as cold exposure, exercise and diet can trigger non-shivering thermogenesis in classical BAT and also browning of WAT, by activating specific signaling cascades within the cell $(20,21)$. For example, cold temperature stimulates the sympathetic nervous system to release norepinephrine, which then binds $\beta_{3}$-adrenergic receptor (15). $\beta_{3}$-adrenergic signaling has a wide range of downstream targets including numerous transcription factors and co-activators that can induce thermogenic genes (22). In addition to the transcriptional regulation, $\beta_{3}$-adrenergic signaling also increases lipolysis and glucose uptake to support thermogenesis (23). In this review, we discuss underlying signaling pathways that regulate thermogenesis in brown and beige adipocytes, as well as browning of WAT.

\section{$\beta_{3}$-ADRENERGIC SIGNALING}

The dominant signaling pathway that governs non-shivering thermogenesis in brown and beige adipocytes is $\beta_{3}$-adrenergic signaling. Upon cold exposure, norepinephrine released from the sympathetic nervous system binds mainly $\beta_{3}$-adrenergic receptor $\left(\beta_{3}-\mathrm{AR}\right)$ to induce adrenergic signaling $(24,25)$ (Figure 1). $\beta_{3^{-}}$ AR coupled with Gs activates AC, which in turn produces cAMP for PKA activation. A variety of downstream targets of PKA, such as p38, CREB and HSL, enhances thermogenesis by inducing thermogenic gene expression and/or mobilizing substrate to fuel thermogenesis $(26,27)$.

p38, a MAP kinase, phosphorylates multiple transcription factors/coregulators, including ATF2 and PGC1 $\alpha$, both of which promote UCP1 transcription (22). In addition, we recently found that $\mathrm{ZC} 3 \mathrm{H} 10$, previously known to bind RNA, is phosphorylated by p38 upon cold, activating the thermogenic gene program in adipocytes (17). Specifically, ZC3H10 binds a distal upstream region of the UCP1 promoter for transcriptional activation. ZC3H10 also activates NRF1 and TFAM, which facilitate mitochondrial biogenesis to increase thermogenic capacity of adipocytes. Thus, transgenic mice overexpressing $\mathrm{ZC} 3 \mathrm{H} 10$ exhibited increased oxygen consumption, higher BAT temperature and reduced body weight while $\mathrm{ZC} 3 \mathrm{H} 10$ knockout mice displayed decreased oxygen consumption, lower BAT temperature and increased body weight. PGC1 $\alpha$, a downstream target of $\mathrm{p} 38$, serves as co-activator for PPAR $\gamma$ and NRF1 to induce expression of UCP1 and TFAM, respectively $(27,28)$. CREB was also shown to bind to the proximal promoter of UCP1 to potentially activate the transcription.

$\beta_{3}$-adrenergic signaling also increases the expression of ZFP516. ZFP516 recruits LSD1, which demethylases $\mathrm{H} 3 \mathrm{~K} 9$ at the proximal region of UCP1 promoter to induce UCP1 transcription. Furthermore, ZFP516 directly interacts with PRDM16 at the UCP1 promoter and potentially at other BAT gene promoters. ZFP516 depletion in vivo resulted in impaired BAT formation with decreased BAT gene expression and increased muscle gene expression in the presumptive BAT depot, while ZFP516 overexpression increased body temperature, oxygen consumption, browning of WAT, leading to lower body weight. Taken together, ZFP516 is required for BAT development in addition to promoting WAT browning.

Adipose thermogenesis can be enhanced by an increased supply of substrates such as FA and glucose. Upon $\beta_{3}$-AR activation, PKA phosphorylates HSL, now known as diacyl glycerol hydrolase, as well as PLIN on lipid droplets to promote lipolysis. FAs thus produced upon lipolysis can undergo $\beta$-oxidation to eventual production of $\mathrm{NADH}$ and FADH for ETC, during UCP1-mediated thermogenesis $(5,29)$. FA also directly binds UCP1 to assist proton influx into the mitochondrial matrix (30). In the fed state, however, FAs as substrates for thermogenesis seem to originate from white adipose tissue lipolysis, although it was also reported that 


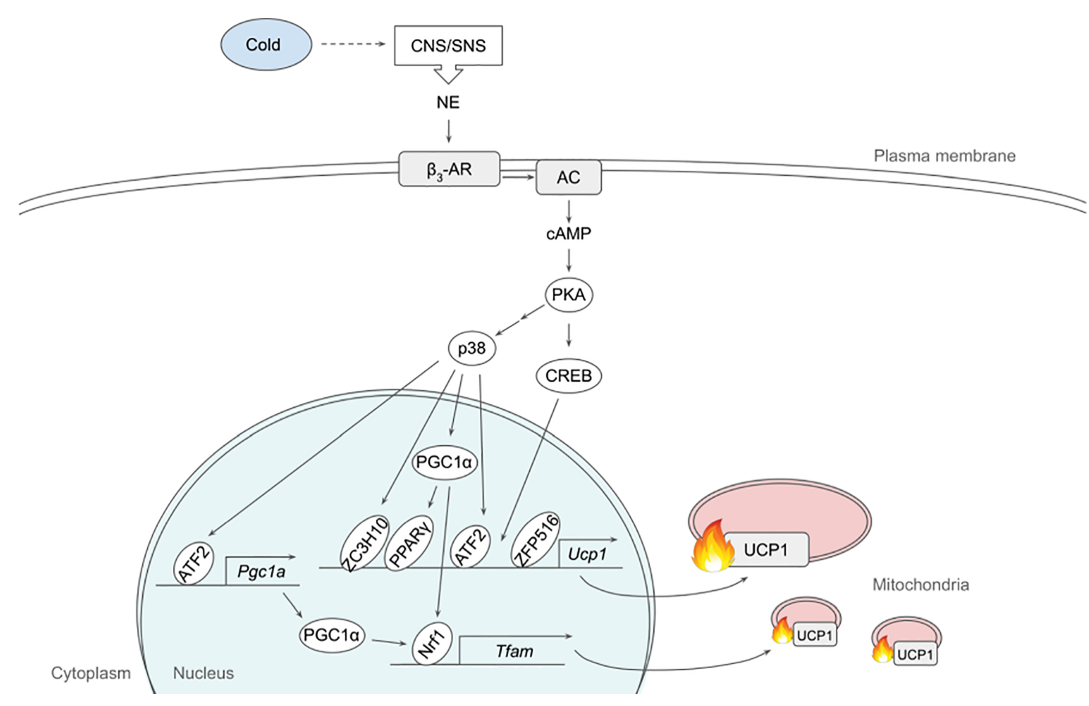

FIGURE 1 | Schematic model of $\beta_{3}$-adrenergic signaling pathway that promotes thermogenesis in adipocytes. Cold stimulates CNS/SNS to secrete NE that binds $\beta_{3}$-AR, which then activates AC producing cAMP. CAMP in turn activates PKA that has a variety of downstream targets, including transcription factors to upregulate thermogenic gene expression. See text for details. AC, adenylate cyclase; ATF2, activating transcription factor $2 ; \beta_{3}-A R, \beta_{3}$-adrenergic receptor; CNS, central nervous system; CREB, CAMP response-element binding protein; ETC, electron transport chain; FFA, free fatty acid; IRF4, interferon regulatory factor 4; NE, norepinephrine; NRF1, nuclear respiratory factor 1; PGC1 $\alpha$, peroxisome proliferator activated receptor $\gamma$ coactivator $\alpha$; PKA, protein kinase A; PPAR $\gamma$, peroxisome proliferator activated receptor $\gamma$; SNS sympathetic nervous system; TCA, tricarboxylic acid; TFAM, mitochondrial transcription factor A; UCP1, uncoupling protein 1; ZC3H10, zinc finger CCCH-type containing 10; ZFP516, zinc finger protein 516.

inhibition of intracellular lipolysis suppressed cold-induced nonshivering thermogenesis in BAT, which was compensated by an increased shivering in humans $(31,32)$. Of note, lipolysis can also be modulated non-adrenergically and this aspect has been extensively reviewed elsewhere (33).

We recently discovered a BAT-specific NADH oxidase, AIFM2, which is required for cold- and diet-induced thermogenesis (34). Upon cold or $\beta_{3}$-adrenergic stimuli, AIFM2 translocates from lipid droplet to the outer side of the inner membrane of mitochondria. AIFM2 oxidizes NADH to NAD, which is needed for glyceraldehyde-3 phosphate dehydrogenase (GAPDH) reaction, in supporting robust glycolysis. In addition, electrons generated from AIFM2 action are transferred to CoQ for ETC activity of mitochondria. AIFM2 KO mice exhibited higher body weight, lower oxygen consumption, and lower BAT temperature whereas AIFM2 overexpression resulted in the opposite phenotypes. In addition, the expression of AIFM2 was induced upon refeeding with high carb diet when examined at thermoneutrality, suggesting that glucose oxidation appears to be especially important in the fed state to promote thermogenesis when the blood glucose level is relatively high.

Collectively, there is no doubt that $\beta_{3}$-AR signaling plays a dominant role in regulating thermogenesis. Besides those mentioned above, there are many more proteins that are involved in and downstream of $\beta_{3}$-AR signaling. Further studies will elucidate more components related to $\beta_{3}$-AR signaling that regulate thermogenesis at various steps such as substrate metabolism, mitochondrial homeostasis, gene expression as well as signal transduction.

\section{INSULIN/IGF1 SIGNALING}

Insulin/IGF1 signaling plays important roles in adipose tissue development and thermogenesis. Mice with fat-specific double knockout of insulin receptor (IR) and IGF1 receptor (IGF1R) (FIGIRKO) exhibited decreased adiposity with more than $85 \%$ reduction in BAT, having lower body weight (35). Indeed, brown preadipocytes from FIGIRKO failed to differentiate in vitro with no increase in the expression of PPAR $\gamma$ and CEBP $\alpha$, indicating that insulin/IGF-1 signaling is critical for BAT development. Since the addition of PPAR $\gamma$ agonist, rosiglitazone, did not rescue the impaired differentiation, insulin/IGF-1 signaling seemed to act on the upstream of PPAR $\gamma / \mathrm{CEBP} \alpha$. Consistently, phosphorylation of CEBP $\beta$ at Thr188, which is required for DNA binding and therefore transcriptional activation of PPAR $\gamma /$ $\mathrm{CEBP} \alpha$, was reduced. FIGIRKO mice were also protected from diet-induced obesity, possibly due to impaired adipose development. FIGIRKO mice failed to maintain their body temperature during cold exposure, although they had higher energy expenditure/basal metabolic rate with no change in activity, this paradox remaining to be explained. In this regard, the study utilized aP2 promoter to express Cre recombinase for gene $\mathrm{KO}$, which could have affected not only adipose tissues but also other cell types, such as macrophages.

The effects of insulin signaling and IGF-1 signaling were further investigated by comparing fat-specific IR single KO (FIRKO) and IGF1R single KO (F-IGFRKO) to IR/IGF1R double $\mathrm{KO}$ (F-IR/IGFRKO), which were all generated by Cre driven by adiponectin promoter (36). F-IGFRKO only had a small 
reduction in both WAT and BAT, while F-IR/IGFRKO had an almost complete loss of adipose tissue. Interestingly, F-IRKO had a large WAT reduction with an unexplainable 50\% increase in BAT with large unilocular lipid droplet. BAT of F-IR/IGFRKO exhibited increased BAT gene expression and decreased WAT gene expression, whereas that of F-IRKO showed no change in BAT or WAT marker gene expression. Regardless, the expression of genes involved in BAT function, including UCP1 and other thermogenic genes, TFAM for example, was reduced in both F-IR/IGFRKO and F-IRKO, which could explain why both F-IR/IGFRKO and F-IRKO failed to maintain body temperature during the cold challenge. Indeed, F-IRKO had reduced mitochondrial content and oxygen consumption.

BAT-specific IR KO mice created by using UCP1 promoter-Cre, however, showed impaired BAT development (37). Since the expression of CEBP $\alpha$, but not PPAR $\gamma$, decreased in BAT of the $\mathrm{KO}$ mice, insulin signaling likely promotes brown adipogenesis mainly through CEBP $\alpha$. Interestingly, UCP1 expression increased significantly in BAT and the $\mathrm{KO}$ mice even had leaner phenotype, possibly due to the remaining BAT compensating for the decreased thermogenesis. Another mechanism where insulin/IGF1 signaling can promote BAT development is by increasing proliferation. Insulin and IGF1, not only stimulated IRS1 and IRS2 tyrosine phosphorylation and their association with PI3K, but also enhanced IRS1-associated GRB2 phosphorylation, which can activate RASMAPK pathway for proliferation (38). BAT-specific IGF1R KO mice, on the other hand, had normal BAT size and normal body weight, while UCP1 expression in BAT was reduced and the KO mice were cold intolerant, suggesting that IGF1 signaling enhances BAT thermogenesis (39).

Insulin/IGF1 signaling plays a role in browning of WAT as well. PTEN antagonizes the action of PI3K by converting PIP3 back to PIP2 and, therefore, insulin/IGF1 signaling. PTEN knockdown (KD) by direct injection of Rec2-Cre in iWAT of PTEN floxed mice increased AKT signaling and induced browning (40). These effects were blocked by administration of an AKT inhibitor, indicating that the PI3K-AKT pathway promotes browning. Transgenic mice overexpressing PTEN were reported to have increased energy expenditure with hyperactivation of BAT. However, PTEN was overexpressed globally in these animals, making it difficult to interpret the exact contribution of PTEN in a tissue-specific manner (41).

Interestingly, insulin signaling can be modulated by $\beta_{3^{-}}$ adrenergic signaling. $\beta_{3}$-adrenergic signaling pretreatment reduced the activation of IR, IRS1 and IRS2. In addition, $\beta_{3^{-}}$ adrenergic signaling mitigated AKT activation and IRS1associated activation of PI3K upon insulin treatment, while it did not affect MAPK activation and IRS2-associated activation of PI3K. Moreover, $\beta_{3}$-adrenergic signaling abolished insulinmediated glucose uptake in brown adipocytes (42). In this regard, cold exposure has also been shown to alter the expression of many molecules involved in the insulin signaling pathway (43). Specifically, the expression of IRS1 and IRS2 as well as phosphorylation of AKT are upregulated. These discrepancies could be due to the differences in the methods used such as in vitro vs in vivo, mouse vs rat as well as protein phosphorylation vs transcriptome.
Taken together, IR/IGF1R signaling promotes adipose tissue thermogenesis by supporting BAT development, browning of WAT and BAT function. Some of those studies mentioned above are even conflicting, owing at least partly to the differences in promoters used to express Cre, which affects not only the efficiency of gene $\mathrm{KO}$, but also tissue specificity. Even with a tissue-specific KO, some phenotypes could manifest because of secondary effects rather than direct, primary effects. Therefore, it would be important to recapitulate the results in vitro as well. Further research is needed to better clarify the role of IR and IGF1R signaling in adipose tissue thermogenesis.

\section{THYROID HORMONE}

Among various ligands/hormones that bind and function via nuclear hormone receptors, thyroid hormone (TH) is classically known to regulate thermogenesis (44). TH receptors (TR), TR $\alpha$ and $\operatorname{TR} \beta$, in adipocytes enhance BAT thermogenesis and WAT browning. TRs are activated by triiodothyronine (T3), an active form of $\mathrm{TH}$ produced from thyroxine (T4) by type II iodothyronine deiodinase (DIO2). Although $\operatorname{TR} \alpha$ and $\operatorname{TR} \beta$ may have overlapping functions, TR $\beta$ seems mainly responsible for UCP1 induction, whereas TR $\alpha$ might be more important for full $\beta_{3}$-adrenergic response. In hypothyroid mice, TR $\beta$-selective ligand, GC-1, restored UCP1 expression to the same extent as T3, suggesting the significant role of TR $\beta$ in UCP1 upregulation (45). However, GC-1-treated mice failed to maintain body temperature during cold exposure, suggesting that UCP1 induction by $\operatorname{TR} \beta$ alone is not sufficient to support thermogenesis. In agreement with this, GC-1 treatment did not restore CAMP production in isolated brown adipocytes of the hypothyroid mice, which suggests the involvement of TR $\alpha$ in $\beta_{3^{-}}$adrenergic response. The same group later showed that hypothyroid mice with a dominant negative $\operatorname{TR} \beta$ mutation failed to increase UCP1 expression in BAT (46). These hypothyroid TR $\beta$ mutant mice also had reduced cAMP production in BAT and lower body temperature upon $\mathrm{T} 3$ replacement and $\beta_{3}$-adrenergic stimulation, implying that TR $\beta$ might at least partly participate in $\beta_{3}$-adrenergic response. Although it has also been reported that TR $\alpha$ could bind UCP1 enhancer region for transcriptional activation, the results of the study were relied on in vitro experiments as opposed to the previously mentioned studies that investigated its role in animal models (47). DIO2 is another important TH/TR target that contributes to thermogenesis. GC-1 upregulated DIO2 mRNA and enzyme activity upon $\beta_{3}$-adrenergic stimulation, while TR $\alpha$ selective ligand, CO23, increased DIO2 mRNA expression only (48). Indeed, DIO2 KO brown adipocytes exhibited reduced UCP1 expression, oxygen consumption and lipolysis in response to $\beta 3$-adrenergic stimuli, which was reversed by $\mathrm{T} 3$ administration (49).

TH/TR can enhance BAT thermogenesis through regulation of mitochondrial homeostasis as well (50). Hyperthyroid mice showed increased expression of mitochondrial proteins including COX4I1 and TOMM20 and increased mitochondrial DNA copy number in BAT, implying that $\mathrm{TH}$ promoted mitochondrial biogenesis. On the 
other hand, T3-treated brown adipocytes exhibited mitochondrial remnants inside autophagic vesicles, suggesting that T3 induced mitophagy. Inhibition of autophagy/mitophagy in hyperthyroid mice indeed increased mitochondrial proteins. Mitochondrial turnover was further assessed using MitoTimer, which showed upregulation of both clearance of mature mitochondria and generation of new mitochondria by T3 treatment. The importance of mitochondrial turnover for thermogenesis was confirmed by suppression of autophagy/mitophagy by ATG5 KD, which decreased oxygen consumption in brown adipocytes, and also conditional ATG5 $\mathrm{KO}$ in hyperthyroid mice that showed reduced body temperature.

T3 treatment has been shown to upregulate UCP1 and mitochondria-related genes in white adipocytes as well, by increasing mitochondrial biogenesis $(51,52)$. This was accompanied by elevated oxygen consumption and FA oxidation, indicating the role of T3 in WAT browning. Similar to that observed in brown adipocytes, UCP1 upregulation in WAT was mediated by TR $\beta$ rather than TR $\alpha$ since hyperthyroid TR $\beta$ KO mice failed to increase UCP1 expression in WAT (53). In addition to directly upregulating UCP1 transcription, T3 was reported also to trigger AMPK activation, which in turn activated the p38/ATF2 pathway to promote UCP1 expression in WAT, although the mechanism by which T3 could activate AMPK remains to be addressed (54).

Recently, a TH metabolite, 3,5 Diiodo-L-Thyronine (T2), was reported to affect thermogenesis as well (55). T2 administration restored lipid morphology, vascularization, innervation and mitochondrial content/activity in BAT of hypothyroid rats, which were accompanied by increased expression of UCP1 and PGC1 $\alpha$, leading to improved thermogenesis (56). Similarly, T2 seems to promote WAT browning in high fat diet-induced obese rats partly through upregulation of PRDM16 (57). Overall, it can be concluded that the TH/TR promotes BAT thermogenesis and WAT browning.

\section{TRANSFORMING GROWTH FACTOR $\beta$ SUPERFAMILY}

Multiple members of the transforming growth factor beta (TGF $\beta$ ) superfamily have been shown to regulate thermogenesis (Figure 2). In fact, bone morphogenetic protein 7 (BMP7), was reported first to trigger commitment of mesenchymal progenitor cells to brown adipocyte lineage by activating SMAD1/5/8 (58). Without the canonical differentiation induction cocktail, BMP7 induced the expression of both adipogenic and brown-adipocyte specific genes, such as CEBPs, PPAR $\gamma$, PRDM16, PGC1 $\alpha$, and UCP1, while downregulating adipogenic suppressors such as Pref-1 and WNT10A. BMP7 KO mice exhibited reduced BAT mass that had almost undetectable UCP1 expression. In contrast, BMP7 overexpression via adenovirus injection increased the expression of PRDM16 and UCP1 in BAT, energy expenditure and body temperature, while decreasing body weight. In mature brown adipocytes, BMP7 increased mitochondrial activity through p38-ATF2 and SMAD1/5/8 pathways (59). BMP7- treated cells upregulated the expression of CD36 and CPT1 that transport FAs into the cells and mitochondria, respectively, resulting in increased citrate synthase activity and FA oxidation. Administration of BMP7 in vivo increased the expression of CD36 in BAT, which was accompanied by increased oxygen consumption. In addition, respiratory exchange ratio in these animals decreased, reflecting the substrate utilization switching towards fatty acids. Another study also confirmed the similar effects of BMP7, where BMP7 administration increased the expression of UCP1 and CD36 in BAT, BAT mass, energy expenditure and fat oxidation (60). Furthermore, these authors found that BMP7 induced the expression of BAT genes in WAT depots with overall reduced WAT size, suggesting that BMP7 promotes browning of WAT. This is supported by a finding that demonstrated BMP7 driving human adipogenic stem cells into beige adipocytes (61). BMP7 treatment promoted the expression of beige markers, such as CD137 and TMEM26, in addition to the expression of thermogenic genes, such as UCP1, CIDEA and DIO2.

BMP8b was found to be highly expressed in BAT and its expression increased during differentiation and during cold exposure (62). BMP8b KO mice exhibited lower energy expenditure, lower body temperature and higher body weight, which were exacerbated when these mice were on high fat diet (HFD). Although BMP8b KO mice showed normal BAT size and BAT gene expression, activation of thermogenic signaling pathways examined by phosphorylation of SMAD1/5/8/, p38 and CREB were impaired, indicating that $\mathrm{BMP} 8 \mathrm{~b}$ is indispensable for thermogenic activation of BAT, but not for BAT development. Consistently, BMP8b treatment increased phosphorylation of these proteins in differentiated brown adipocytes, as well as that of HSL and AMPK, resulting in increased lipolysis measured by glycerol release. In addition, BMP8b promoted the activation of AMPK in the central nervous system as well, increasing sympathetic output to BAT to support thermogenesis. In this regard, BMP8b was shown to stimulate sympathetic innervation and vascularization through NRG4 and VEGF, respectively, in both BAT and WAT as well as browning of WAT (63). Mice overexpressing BMP8b showed higher energy expenditure, lower body weight and fat mass, accompanied by decreased thermogenic gene expression in WAT that exhibited multilocular lipid droplets, whereas loss of BMP8b resulted in decreased nerve density.

TGF $\beta /$ SMAD3 signaling pathway has been reported to suppress browning of WAT (64). TGF $\beta$ treatment suppressed activation of PGC1 $\alpha$ promoter-reporter in 3T3-L1 cells, which was abolished by SMAD3 KD, indicating that TGF $\beta$ represses PGC1 $\alpha$ transcription through SMAD3. SMAD3 KO animals had higher body temperature, lower body weight, lower blood glucose, and better response to insulin. WAT of SMAD3 KO mice indeed expressed thermogenic genes, such as UCP1, and exhibited multilocular morphology. Similarly, adipose-specific TGF $\beta$ receptor 1 (TGF $\beta \mathrm{R} 1$ ) KO induced browning, as evidenced by increased UCP1 expression in WAT on HFD, which was accompanied by lower body weight (65). TGFßR1 inhibitor, SB431542, induced brown and beige genes during differentiation of WAT SVF cells, whereas T $\beta R 1$ downregulated 


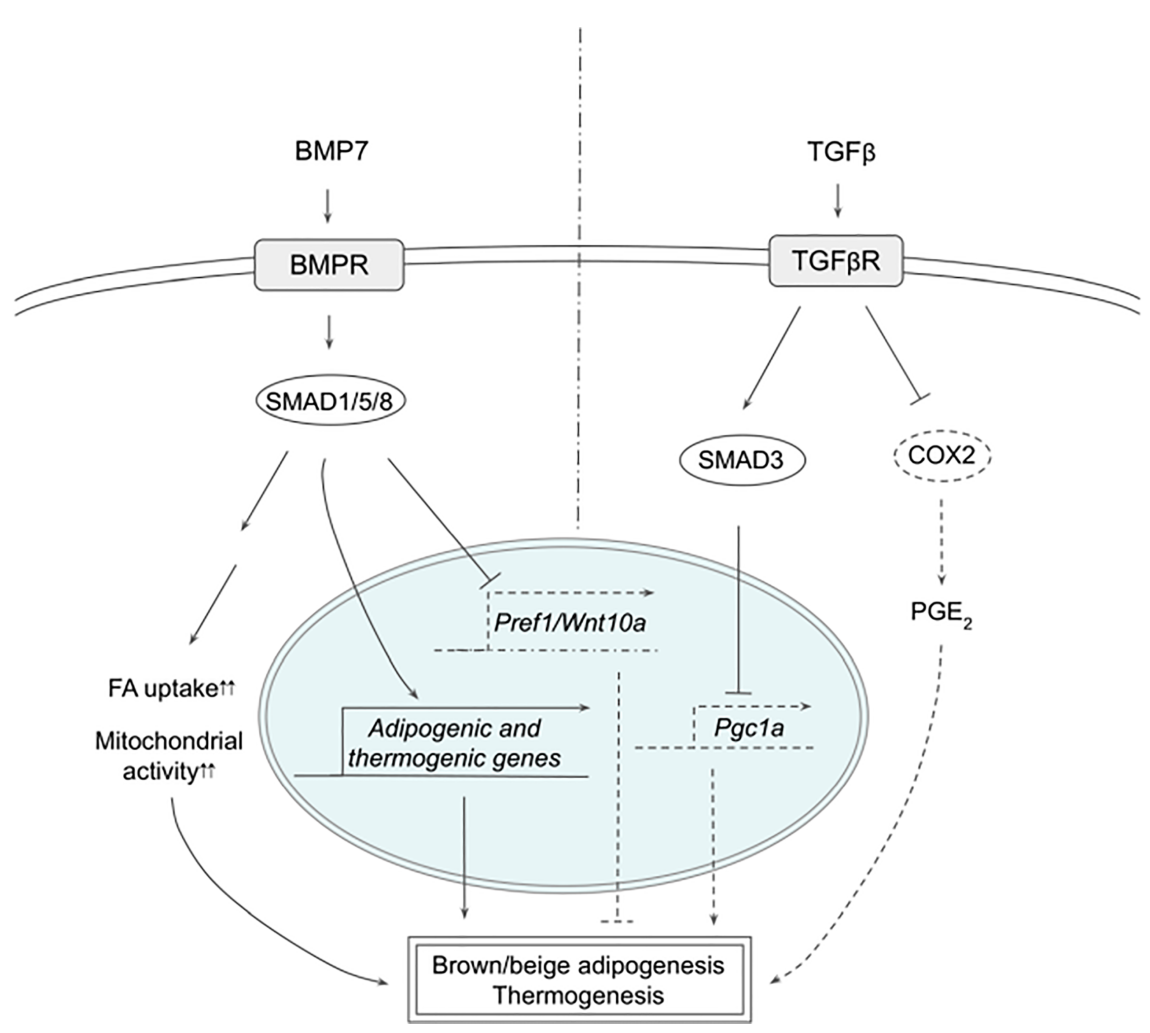

FIGURE 2 | Schematic models of BMP7 and TGF $\beta$ signaling pathways in thermogenesis. BMP7 binds TGF $\beta$ R, which activates SMAD1/5/8, leading to expression of adipogenic and thermogenic genes as well as suppression of Pref1 and Wnt10a in precursor cells to promote brown and beige adipogenesis. In mature adipocytes, BMP7 signaling increases FA uptake and mitochondrial activity, resulting in enhanced thermogenesis. TGF $\beta$ activates SMAD2/3 that suppresses PGC1 $\alpha$ expression and COX2/PGE2 pathway to reduce thermogenesis. See text for details. BMP7, bone morphogenetic protein 7; COX2, cyclooxygenase 2; FA, fatty acid; PGC1 $\alpha$, peroxisome proliferator activated receptor $\gamma$; PGE2, prostaglandin E2; PREF1, preadipocyte factor 1; SMAD, mothers against decapentaplegic homolog; T $\beta R 1$, TGF $\beta$ receptor 1; TGF $\beta$, Transforming growth factor beta; TGF $\beta$ R, TGF $\beta$ receptor; WNT10a, Wnt family member 10A.

those genes. Interestingly, WAT of TGFßR1 KO also increased the expression of genes involved in prostaglandin pathways, such as COX2, implying that prostaglandin pathway might be involved in browning of WAT. SMAD3 KD in 3T3-L1 cells increased the expression of COX2 and other prostaglandin pathway-related proteins. Treatment with $\mathrm{PGE}_{2}$, a product of $\mathrm{COX} 2$, upregulated thermogenic gene expression, such as CIDEA1 and PGC1 $\alpha$, in the presumptive beige precursor cells, which was further augmented by the addition of SB431542, implying the possible interaction between TGF $\beta / S M A D 3$ and $\mathrm{COX} 2 / \mathrm{PGE}_{2}$ pathways. These presumptive beige precursor cells were isolated and selected from WAT by FACS using a series of cell surface markers and were termed inducible beige stem/progenitor cells (iBSCs). iBSCs were then transplanted subcutaneously into immunodeficient nude mice on HFD with IPTT-300 transponder. During the cold exposure, iBSCs from T $\beta R 1$ KO mice maintained higher temperature and showed higher expression of thermogenic genes with multilocular lipid droplet morphology, suggesting that the KO iBSCs underwent beige adipogenesis. Altogether, TGF $\beta / S M A D 3$ signaling negatively regulates WAT browning although further research will help to clarify the contributions of different downstream pathways.

\section{CONCLUDING REMARK}

The discovery of brown or brown-like adipocytes in human adults has made targeting adipocytes an attractive strategy to fight against obesity and its associated diseases. Activation of existing thermogenic adipocytes and/or induction of new thermogenic adipocytes can be mediated by various hormones and their signaling cascades, including those important pathways we describe in this review. Understanding the precise molecular mechanisms underlying regulation of thermogenesis and browning of WAT is critical for advancement of the future anti-obesity therapeutics.

\section{AUTHOR CONTRIBUTIONS}

CT drafted and HS revised the manuscript. All authors contributed to the article and approved the submitted version.

\section{FUNDING}

The work was supported in part by DK120075 to HSS. 


\section{REFERENCES}

1. Nedergaard J, Cannon B. Brown adipose tissue as a heat-producing thermoeffector. Handb Clin Neurol (2018) 156:137-52. doi: 10.1016/B9780-444-63912-7.00009-6

2. Lidell ME. Brown Adipose Tissue in Human Infants. In: A Pfeifer, M Klingenspor, S Herzig, editors. Brown Adipose Tissue. Cham: Springer International Publishing (2019). p. 107-23.

3. Wang Q, Zhang M, Xu M, Gu W, Xi Y, Qi L, et al. Brown adipose tissue activation is inversely related to central obesity and metabolic parameters in adult human. PloS One (2015) 10(4):e0123795. doi: 10.1371/journal. pone. 0123795

4. Saito M. Brown adipose tissue as a regulator of energy expenditure and body fat in humans. Diabetes Metab J (2013) 37(1):22-9. doi: 10.4093/ dmj.2013.37.1.22

5. Fenzl A, Kiefer FW. Brown adipose tissue and thermogenesis. Hormone Mol Biol Clin Invest (2014) 19(1):1868-91. doi: 10.1515/hmbci-2014-0022

6. Lattin JE, Schroder K, Su AI, Walker JR, Zhang J, Wiltshire T, et al. Expression analysis of G Protein-Coupled Receptors in mouse macrophages. Immunome Res (2008) 4:5. doi: 10.1186/1745-7580-4-5

7. Chouchani ET, Kazak L, Spiegelman BM. New Advances in Adaptive Thermogenesis: UCP1 and Beyond. Cell Metab (2018) 29(1):27-37. doi: 10.1016/j.cmet.2018.11.002

8. Herz CT, Kiefer FW. Adipose tissue browning in mice and humans. J Endocrinol (2019) 241(3):R97-109. doi: 10.1530/JOE-18-0598

9. Wu J, Boström P, Sparks LM, Ye L, Choi JH, Giang A-H, et al. Beige adipocytes are a distinct type of thermogenic fat cell in mouse and human. Cell (2012) 150(2):366-76. doi: 10.1016/j.cell.2012.05.016

10. Shao M, Wang QA, Song A, Vishvanath L, Busbuso NC, Scherer PE, et al. Cellular Origins of Beige Fat Cells Revisited. Diabetes (2019) 68(10):1874-85. doi: $10.2337 / \mathrm{db} 19-0308$

11. Wang W, Seale P. Control of brown and beige fat development. Nat Rev Mol Cell Biol (2016) 17(11):691-702. doi: 10.1038/nrm.2016.96

12. Rajakumari S, Wu J, Ishibashi J, Lim H-W, Giang A-H, Won K-J, et al. EBF2 determines and maintains brown adipocyte identity. Cell Metab (2013) 17 (4):562-74. doi: 10.1016/j.cmet.2013.01.015

13. Seale P, Bjork B, Yang W, Kajimura S, Chin S, Kuang S, et al. PRDM16 controls a brown fat/skeletal muscle switch. Nature (2008) 454(7207):961-7. doi: 10.1038/nature07182

14. Park JH, Kang HJ, Kang SI, Lee JE, Hur J, Ge K, et al. A multifunctional protein, EWS, is essential for early brown fat lineage determination. Dev Cell (2013) 26(4):393-404. doi: 10.1016/j.devcel.2013.07.002

15. Inagaki T, Sakai J, Kajimura S. Transcriptional and epigenetic control of brown and beige adipose cell fate and function. Nat Rev Mol Cell Biol (2016) 17(8):480-95. doi: 10.1038/nrm.2016.62

16. Dempersmier J, Sambeat A, Gulyaeva O, Paul SM, Hudak CSS, Raposo HF, et al. Cold-inducible Zfp516 activates UCP1 transcription to promote browning of white fat and development of brown fat. Mol Cell (2015) 57 (2):235-46. doi: 10.1016/j.molcel.2014.12.005

17. Yi D, Dempersmier JM, Nguyen HP, Viscarra JA, Dinh J, Tabuchi C, et al. Zc3h10 Acts as a Transcription Factor and Is Phosphorylated to Activate the Thermogenic Program. Cell Rep (2019) 29(9):2621-33.e4. doi: 10.1016/ j.celrep.2019.10.099

18. Koppen A, Kalkhoven E. Brown vs white adipocytes: the PPARgamma coregulator story. FEBS Lett (2010) 584(15):3250-9. doi: 10.1016/ j.febslet.2010.06.035

19. Kajimura S, Seale P, Kubota K, Lunsford E, Frangioni JV, Gygi SP, et al. Initiation of myoblast to brown fat switch by a PRDM16-C/EBP-beta transcriptional complex. Nature (2009) 460(7259):1154-8. doi: 10.1038/ nature 08262

20. Gill JA, La Merrill MA. An emerging role for epigenetic regulation of Pgc- $1 \alpha$ expression in environmentally stimulated brown adipose thermogenesis. Environ Epigenet (2017) 3(2):dvx009. doi: 10.1093/eep/dvx009

21. Ikeda K, Maretich P, Kajimura S. The Common and Distinct Features of Brown and Beige Adipocytes. Trends Endocrinol Metab (2018) 29(3):191-200. doi: 10.1016/j.tem.2018.01.001

22. Yi D, Nguyen HP, Sul HS. Epigenetic dynamics of the thermogenic gene program of adipocytes. Biochem J (2020) 477(6):1137-48. doi: 10.1042/ BCJ20190599
23. Reverte-Salisa L, Sanyal A, Pfeifer A. Brown Adipose Tissue: Role of cAMP and cGMP Signaling in Brown Fat. In: A Pfeifer, M Klingenspor, S Herzig, editors. Brown Adipose Tissue. Cham: Springer International Publishing (2019). p. 161-82.

24. Liu J, Wang Y, Lin L. Small molecules for fat combustion: targeting obesity. Acta Pharm Sin B (2019) 9(2):220-36. doi: 10.1016/j.apsb.2018.09.007

25. Schena G, Caplan MJ. Everything You Always Wanted to Know about $\beta 3$-AR ${ }^{*}\left({ }^{*}\right.$ But Were Afraid to Ask). Cells (2019) 8(4):357. doi: 10.3390/cells8040357

26. Shi F, Collins S. Second messenger signaling mechanisms of the brown adipocyte thermogenic program: an integrative perspective. Hormone Mol Biol Clin Invest (2017) 31(2):1868-91. doi: 10.1515/hmbci-2017-0062

27. Cao W, Daniel KW, Robidoux J, Puigserver P, Medvedev AV, Bai X, et al. p38 mitogen-activated protein kinase is the central regulator of cyclic AMPdependent transcription of the brown fat uncoupling protein 1 gene. Mol Cell Biol (2004) 24(7):3057-67. doi: 10.1128/MCB.24.7.3057-3067.2004

28. Liang H, Ward WF. PGC-1alpha: a key regulator of energy metabolism. Adv Physiol Educ (2006) 30(4):145-51. doi: 10.1152/advan.00052.2006

29. Symonds ME. Adipose Tissue Biology. Cham: Springer (2017).

30. Kajimura S, Saito M. A new era in brown adipose tissue biology: molecular control of brown fat development and energy homeostasis. Annu Rev Physiol (2014) 76:225-49. doi: 10.1146/annurev-physiol-021113-170252

31. Blondin DP, Frisch F, Phoenix S, Guérin B, Turcotte ÉE, Haman F, et al. Inhibition of Intracellular Triglyceride Lipolysis Suppresses Cold-Induced Brown Adipose Tissue Metabolism and Increases Shivering in Humans. Cell Metab (2017) 25(2):438-47. doi: 10.1016/j.cmet.2016.12.005

32. Shin H, Ma Y, Chanturiya T, Cao Q, Wang Y, Kadegowda AKG, et al. Lipolysis in Brown Adipocytes Is Not Essential for Cold-Induced Thermogenesis in Mice. Cell Metab (2017) 26(5):764-77.e5. doi: 10.1016/ j.cmet.2017.09.002

33. Braun K, Oeckl J, Westermeier J, Li Y, Klingenspor M. Non-adrenergic control of lipolysis and thermogenesis in adipose tissues. J Exp Biol (2018) 221(Pt Suppl 1):jeb165381. doi: 10.1242/jeb.165381

34. Nguyen HP, Yi D, Lin F, Viscarra JA, Tabuchi C, Ngo K, et al. Aifm2, a NADH Oxidase, Supports Robust Glycolysis and Is Required for Cold- and DietInduced Thermogenesis. Mol Cell (2020) 77(3):600-17.e4. doi: 10.1016/ j.molcel.2019.12.002

35. Boucher J, Mori MA, Lee KY, Smyth G, Liew CW, Macotela Y, et al. Impaired thermogenesis and adipose tissue development in mice with fat-specific disruption of insulin and IGF-1 signalling. Nat Commun (2012) 3:902. doi: 10.1038/ncomms 1905

36. Boucher J, Softic S, El Ouaamari A, Krumpoch MT, Kleinridders A, Kulkarni RN, et al. Differential Roles of Insulin and IGF-1 Receptors in Adipose Tissue Development and Function. Diabetes (2016) 65(8):2201-13. doi: $10.2337 / \mathrm{db} 16-0212$

37. Guerra C, Navarro P, Valverde AM, Arribas M, Brüning J, Kozak LP, et al. Brown adipose tissue-specific insulin receptor knockout shows diabetic phenotype without insulin resistance. J Clin Invest (2001) 108(8):1205-13. doi: $10.1172 / \mathrm{JCI} 13103$

38. Valverde AM, Lorenzo M, Pons S, White MF, Benito M. Insulin receptor substrate (IRS) proteins IRS-1 and IRS-2 differential signaling in the insulin/ insulin-like growth factor-I pathways in fetal brown adipocytes. Mol Endocrinol (1998) 12(5):688-97. doi: 10.1210/mend.12.5.0106

39. Viana-Huete V, Guillén C, García-Aguilar A, García G, Fernández S, Kahn CR, et al. Essential Role of IGFIR in the Onset of Male Brown Fat Thermogenic Function: Regulation of Glucose Homeostasis by Differential Organ-Specific Insulin Sensitivity. Endocrinology (2016) 157(4):1495-511. doi: 10.1210/en.2015-1623

40. Huang W, Queen NJ, McMurphy TB, Ali S, Cao L. Adipose PTEN regulates adult adipose tissue homeostasis and redistribution via a PTEN-leptin-sympathetic loop. Mol Metab (2019) 30:48-60. doi: 10.1016/j.molmet.2019.09.008

41. Ortega-Molina A, Efeyan A, Lopez-Guadamillas E, Muñoz-Martin M, Gómez-López $\mathrm{G}$, Cañamero $\mathrm{M}$, et al. Pten positively regulates brown adipose function, energy expenditure, and longevity. Cell Metab (2012) 15 (3):382-94. doi: 10.1016/j.cmet.2012.02.001

42. Klein J, Fasshauer M, Ito M, Lowell BB, Benito M, Kahn CR. $\beta 3$-Adrenergic Stimulation Differentially Inhibits Insulin Signaling and Decreases Insulininduced Glucose Uptake in Brown Adipocytes. J Biol Chem (1999) 274 (49):34795-802. doi: 10.1074/jbc.274.49.34795 
43. Wang X, Wahl R. Responses of the insulin signaling pathways in the brown adipose tissue of rats following cold exposure. PloS One (2014) 9(6):e99772. doi: 10.1371/journal.pone.0099772

44. Yau WW, Yen PM. Thermogenesis in Adipose Tissue Activated by Thyroid Hormone. Int J Mol Sci (2020) 21(8):3020. doi: 10.3390/ijms21083020

45. Ribeiro MO, Carvalho SD, Schultz JJ, Chiellini G, Scanlan TS, Bianco AC, et al. Thyroid hormone-sympathetic interaction and adaptive thermogenesis are thyroid hormone receptor isoform-specific. J Clin Invest (2001) 108 (1):97-105. doi: 10.1172/JCI200112584

46. Ribeiro MO, Bianco SDC, Kaneshige M, Schultz JJ, Cheng S-Y, Bianco AC, et al. Expression of uncoupling protein 1 in mouse brown adipose tissue is thyroid hormone receptor-beta isoform specific and required for adaptive thermogenesis. Endocrinology (2010) 151(1):432-40. doi: 10.1210/en.2009-0667

47. Chen W, Yang Q, Roeder RG. Dynamic interactions and cooperative functions of PGC-1alpha and MED1 in TRalpha-mediated activation of the brown-fat-specific UCP-1 gene. Mol Cell (2009) 35(6):755-68. doi: 10.1016/j.molcel.2009.09.015

48. Martinez de Mena R, Scanlan TS, Obregon M-J. The T3 receptor beta1 isoform regulates UCP1 and D2 deiodinase in rat brown adipocytes. Endocrinology (2010) 151(10):5074-83. doi: 10.1210/en.2010-0533

49. de Jesus LA, Carvalho SD, Ribeiro MO, Schneider M, Kim SW, Harney JW, et al. The type 2 iodothyronine deiodinase is essential for adaptive thermogenesis in brown adipose tissue. J Clin Invest (2001) 108(9):1379-85. doi: 10.1172/JCI200113803

50. Yau WW, Singh BK, Lesmana R, Zhou J, Sinha RA, Wong KA, et al. Thyroid hormone (T3) stimulates brown adipose tissue activation via mitochondrial biogenesis and MTOR-mediated mitophagy. Autophagy (2019) 15(1):131-50. doi: $10.1080 / 15548627.2018 .1511263$

51. Lee J-Y, Takahashi N, Yasubuchi M, Kim Y-I, Hashizaki H, Kim M-J, et al. Triiodothyronine induces UCP-1 expression and mitochondrial biogenesis in human adipocytes. Am J Physiol Cell Physiol (2012) 302(2):C463-72. doi: 10.1152/ajpcell.00010.2011

52. Krause K. Novel Aspects of White Adipose Tissue Browning by Thyroid Hormones. Exp Clin Endocrinol Diabetes (2020) 128(6-07):446-9. doi: 10.1055/a-1020-5354

53. Johann K, Cremer AL, Fischer AW, Heine M, Pensado ER, Resch J, et al. Thyroid-Hormone-Induced Browning of White Adipose Tissue Does Not Contribute to Thermogenesis and Glucose Consumption. Cell Rep (2019) 27 (11):3385-400.e3. doi: 10.1016/j.celrep.2019.05.054

54. Matesanz N, Bernardo E, Acín-Pérez R, Manieri E, Pérez-Sieira S, HernándezCosido L, et al. MKK6 controls T3-mediated browning of white adipose tissue. Nat Commun (2017) 8(1):856. doi: 10.1038/s41467-017-00948-z

55. Louzada RA, Carvalho DP. Similarities and Differences in the Peripheral Actions of Thyroid Hormones and Their Metabolites. Front Endocrinol (2018) 9:394. doi: 10.3389/fendo.2018.00394

56. Lombardi A, Senese R, De Matteis R, Busiello RA, Cioffi F, Goglia F, et al. 3,5Diiodo-L-thyronine activates brown adipose tissue thermogenesis in hypothyroid rats. PloS One (2015) 10(2):e0116498. doi: 10.1371/ journal.pone. 0116498

57. Senese R, Cioffi F, De Matteis R, Petito G, de Lange P, Silvestri E, et al. 3,5 Diiodo-l-Thyronine $\left(\mathrm{T}_{2}\right)$ Promotes the Browning of White Adipose Tissue in High-Fat Diet-Induced Overweight Male Rats Housed at Thermoneutrality. Cells (2019) 8(3):256. doi: 10.3390/cells8030256

58. Tseng Y-H, Kokkotou E, Schulz TJ, Huang TL, Winnay JN, Taniguchi CM, et al. New role of bone morphogenetic protein 7 in brown adipogenesis and energy expenditure. Nature (2008) 454(7207):1000-4. doi: 10.1038/ nature 07221

59. Townsend KL, An D, Lynes MD, Huang TL, Zhang H, Goodyear LJ, et al. Increased mitochondrial activity in BMP7-treated brown adipocytes, due to increased CPT1- and CD36-mediated fatty acid uptake. Antioxid Redox Signal (2013) 19(3):243-57. doi: 10.1089/ars.2012.4536

60. Boon MR, van den Berg SAA, Wang Y, van den Bossche J, Karkampouna S, Bauwens M, et al. BMP7 activates brown adipose tissue and reduces dietinduced obesity only at subthermoneutrality. PloS One (2013) 8(9):e74083. doi: 10.1371/journal.pone. 0074083

61. Okla M, Ha J-H, Temel RE, Chung S. BMP7 drives human adipogenic stem cells into metabolically active beige adipocytes. Lipids (2015) 50(2):111-20. doi: 10.1007/s11745-014-3981-9

62. Whittle AJ, Carobbio S, Martins L, Slawik M, Hondares E, Vázquez MJ, et al. BMP8B increases brown adipose tissue thermogenesis through both central and peripheral actions. Cell (2012) 149(4):871-85. doi: 10.1016/ j.cell.2012.02.066

63. Pellegrinelli V, Peirce VJ, Howard L, Virtue S, Türei D, Senzacqua M, et al. Adipocyte-secreted BMP8b mediates adrenergic-induced remodeling of the neuro-vascular network in adipose tissue. Nat Commun (2018) 9(1):4974. doi: 10.1038/s41467-018-07453-x

64. Yadav H, Quijano C, Kamaraju AK, Gavrilova O, Malek R, Chen W, et al. Protection from obesity and diabetes by blockade of TGF- $\beta /$ Smad 3 signaling. Cell Metab (2011) 14(1):67-79. doi: 10.1016/j.cmet.2011.04.013

65. Wankhade UD, Lee J-H, Dagur PK, Yadav H, Shen M, Chen W, et al. TGF- $\beta$ receptor 1 regulates progenitors that promote browning of white fat. $\mathrm{Mol}$ Metab (2018) 16:160-71. doi: 10.1016/j.molmet.2018.07.008

Conflict of Interest: The authors declare that the research was conducted in the absence of any commercial or financial relationships that could be construed as a potential conflict of interest.

Copyright $\odot 2021$ Tabuchi and Sul. This is an open-access article distributed under the terms of the Creative Commons Attribution License (CC BY). The use, distribution or reproduction in other forums is permitted, provided the original author $(s)$ and the copyright owner(s) are credited and that the original publication in this journal is cited, in accordance with accepted academic practice. No use, distribution or reproduction is permitted which does not comply with these terms. 